Health and medicine | Dr Jin Woo Choi \& Yi Rang Kim

\section{Using photodynamic therapy to target circulating tumour cells}

\begin{abstract}
Dr Jin Woo Choi from Kyung Hee University \& Yi Rang Kim from Oncocross, Ltd. in South Korea, have developed a novel photodynamic therapy (PDT) method which can eradicate reducing the risk of metastatic cancer development. In PDT light is used to illuminate and activate photosensitisers which then produce potent reactive oxygen species whic can induce cell damage and death. The team combined this phenomenon with the process where energy is transferred from a light activated substrate to a photosensitizer, to selectively target and destroy CTCs and prevent metastasis.
\end{abstract}

due to a lack of efficient technology for their detection. Significant advances in technology have led to improvements in as immunomagnetic separation with antibodies, fibre-optic array scanning technology and microfluidic platforms. Many studies have determined that a high CTC number is associated with poor cancer prognosis. Therefore, it is essential that CTC-specific targeted therapies are developed that eliminate CTCs from the vascular system and improve prognosis. In practice, however, this has been challenging to achieve due to the unpredictable and dynamic nature Kyung Hee University and Dr Kim from
Specific wavelengths of light activate certain photosensitisers which then convert tissue oxygen into reactive oxygen species and free radicals.
A .

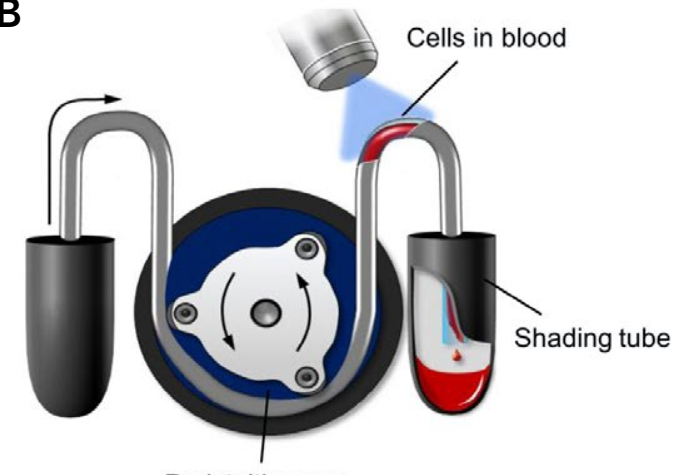

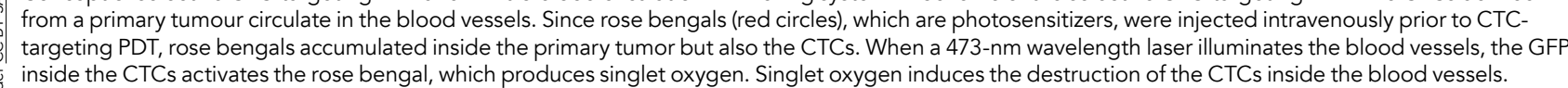

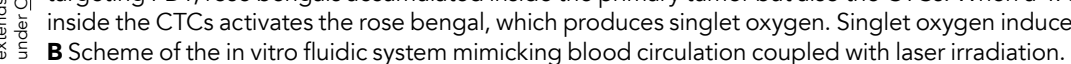

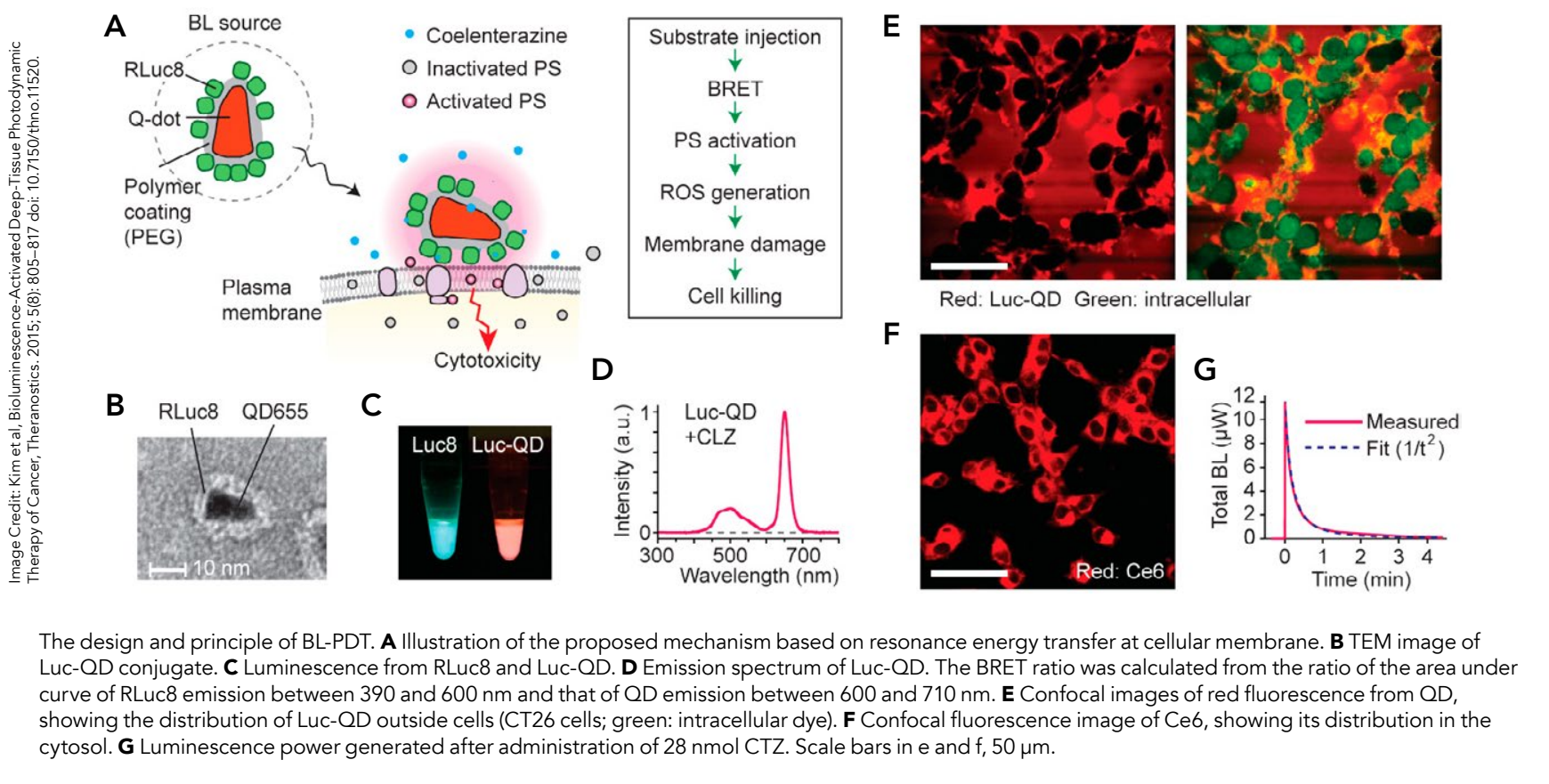

Oncocross, Ltd set out to optimise the ground breaking photodynamic therapy (PDT) to focus on eradicating fluorescent protein-expressing CTCs.

PHOTODYNAMIC THERAPY PDT is a treatment method that uses light and specific drugs known as photosensitisers. Specific wavelengths of light activate certain photosensitisers which then convert tissue oxygen into reactive oxygen species (ROS) and tree radicals. High levels of ROS can cause oxidative stress, damaging surrounding cellular structures, and can even induce PDT apoptosis of cancercells. Although PDThas major biomedical potential, a the activatio lighe is the detivery of Visible light penetrates a few hundred micrometres in tissue, therefore clinical use of light-based techniqu to superficial layers such as the skin and retina. However, there is an interesting solution to this problem - the concept of internal light sources. The implantation of light emitting diodes or fibre-optic light sources are viable, but very invasive for the patient. One attractive alternative to optoelectronic tools is the use of bioluminescence (BL) because the source molecules, such as enzymes, can be delivered with minimal invasion. These bio-luminating molecules can be use in a process called bioluminescence BRET, an enctgy transfer (BRET). In (typically a lucif biseluminescent donor

Around $60 \%$ of GFP-expressing cells were selectively killed when irradiated with $473 \mathbf{~ n m}$ blue light laser after Rose Bengal treatment.

energy to an acceptor. This is a distant-_ using bioluminescence PDT (BL-PDT) dependent process and the range over the foundation of which is the BRET which energy transfer can take place is limited to 10 nanometres. PDT AND BPET To overc Ber the as the acceptor protein, the team To overcome the challenges of using showed that BL-PDT can be used to team investigated the potential of suppress tumour growh in mice for three

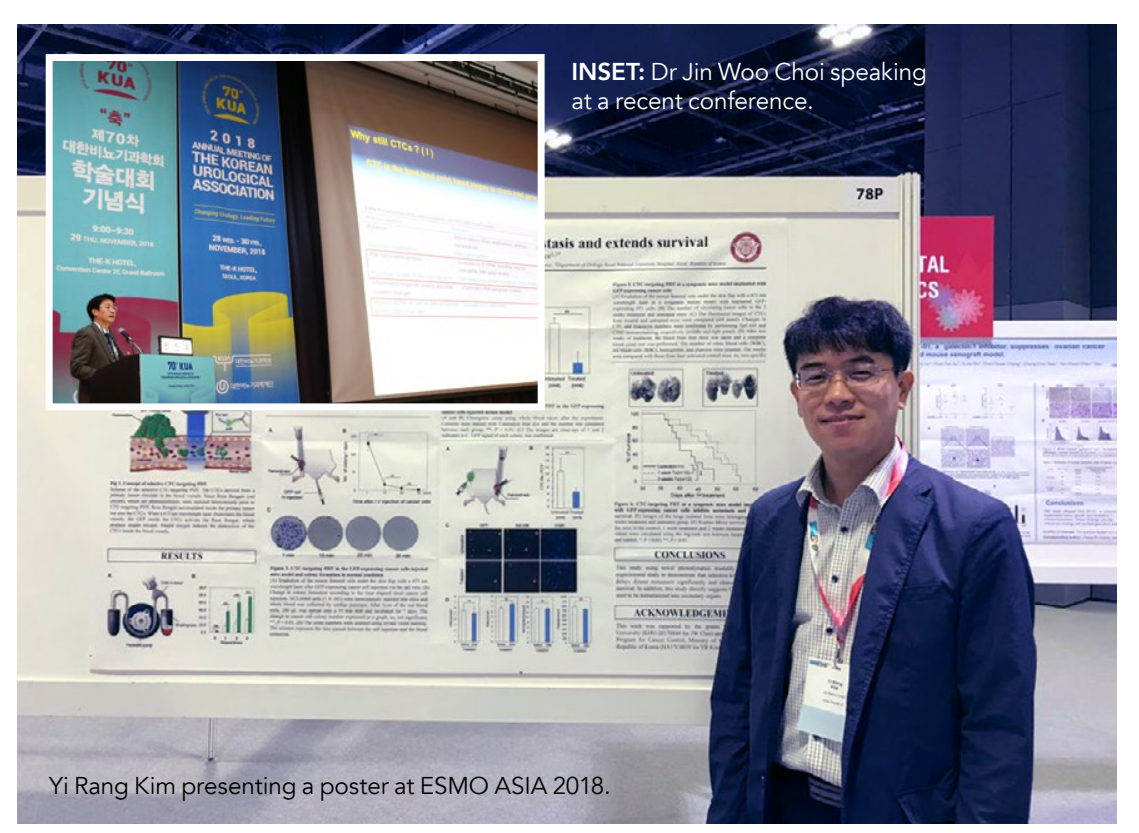



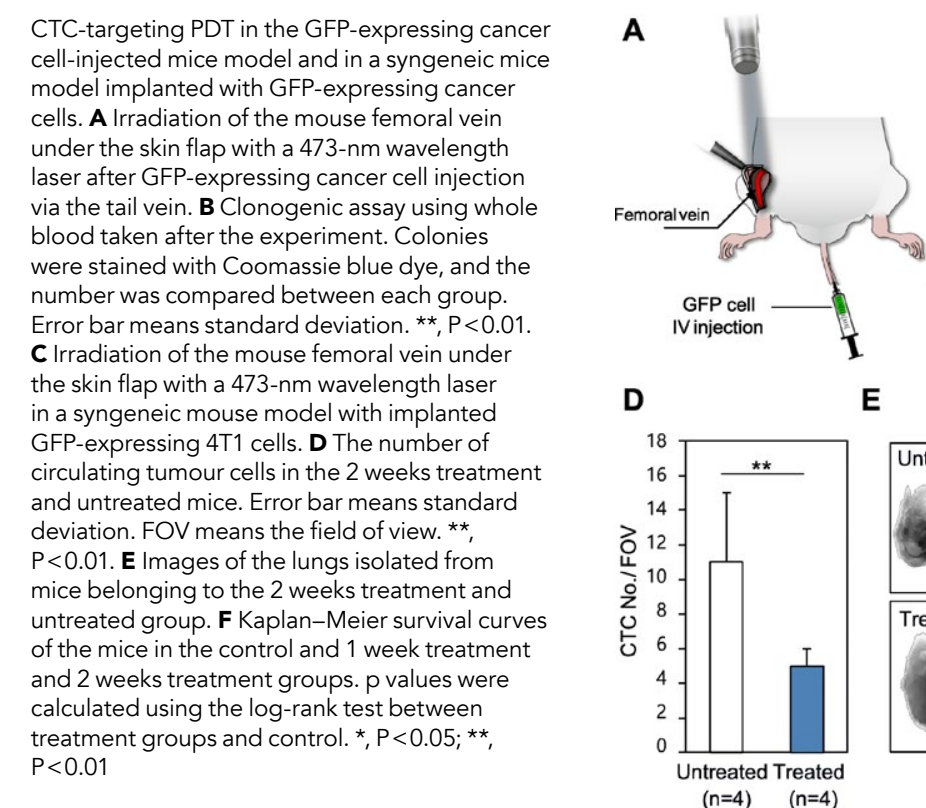

B

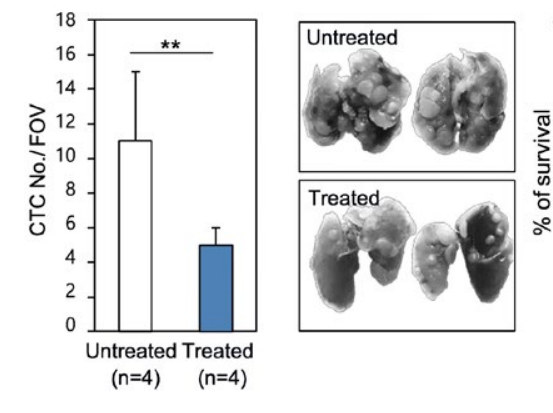

lung cancer and colorectal cancer.

and organelles such as mitochondria

and lysosomes. Activation of $\mathrm{Ce} 6$ by

energy transfer from Rluc8, results

in the generation of ROS, which

subsequently induces lipid peroxidation

and destruction of the cell membrane.

This disrupts intracellular homeostasis

and cell death is induced. The team

demonstrated the effectiveness of BL-

donor fluorophore which transfers its excitation energy to a nearby acceptor molecule. In this study, the team used green fluorescent protein (GFP) as the linker of energy transfer between a specific wavelength light source and the
photosensitiser Rose Bengal. The team used GFP expressing cancer cells to highlight the function of GFP to target a specific cell population based on PDT. The team had to ensure that the laser

Strikingly, the number of lung metastatic nodules in the treated mice was

significantly lower and these mice survived for longer than untreated mice.

PDT for cancer cell ablation in the local lymph nodes, reducing metastatic spread and significantly enhancing animal survival. The lymph nodes are located at depths not accessible by external light illumination, therefore BL-PDT could have huge therapeutic potentia for targeting metastasis.

PDT AND FRET

Dr Choi and Dr Kim decided to extend Deir research by investigating wheth his phenomenon could also be adopted in Foster resonance energy transter (FRT) to specifcally target cancer cells. In RRET, ight ilumination excited GFP but had no effect on the photosensitiser to minimise effects on the emission spectrum of GFP had to overlap with the absorption spectrum of the photosensitiser to ensure that GFP-expressing cells were selectively killed. As a result, the team chose a t73 nm blue laser. The results showed around $60 \%$ of GPP-expressing cells with a 473 n bline lighen ir radiated Bester aster Rose This is due to tessing cells were killed. Bengal treatment. However, only $20 \%$ Rose Be to the production or ROS by
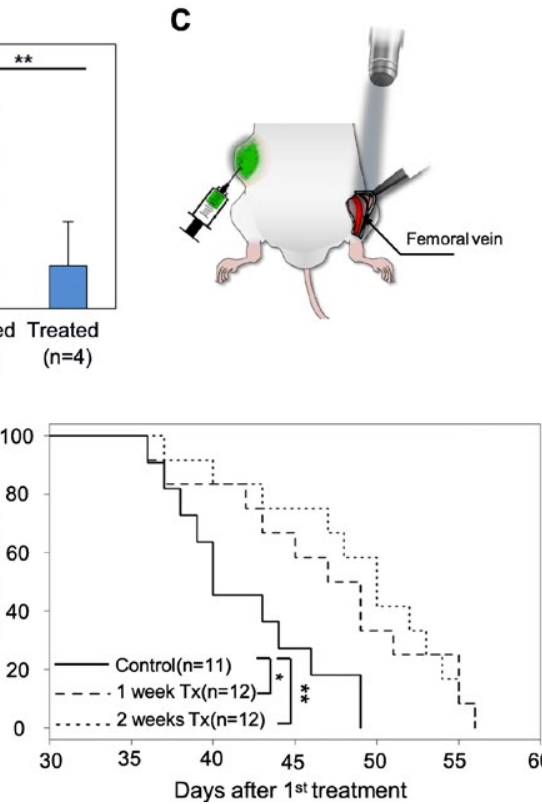

USING FRET FOR CTC ABLATION

The success of the study using FRET

PDT inspired Dr Choi and Dr Kim

to target CTC ablation using GFP

expressed CTCs and Rose Bengal.

The team performed an in vitro study

a peristaltic pump to mimic circulation

within the blood vessels. GFP-expressing

CTCs and GFP-non-expressing CTCs

were incubated with Rose Bengal and

were passed through the tubing. Results

showed that cell damage and death was greater among GFP-expressing CTCs An pared to GFP-non-expressing CTCs. An in vivo study was also conducted: bose Bengal into mpessing CTCs and then illuminated onto the mouse femor vein. Results showed that the number of treated CTC colonies dramatically decreased. Additionally CTC-targeting PDT was performed in mice with GFPexpressing metastatic cells transplanted into their flanks. Again, the number of CTC cells in the irradiated mice was significantly lower than the untreated mice. Strikingly, the number of lung metastatic nodules in the treated mice was significantly lower and these mice survived for longer than untreated mice. Excitingly, these results demonstrate that CTC-targeted therapies aimed at improving cancer prognosis may provide a promising new approach for developing personalised

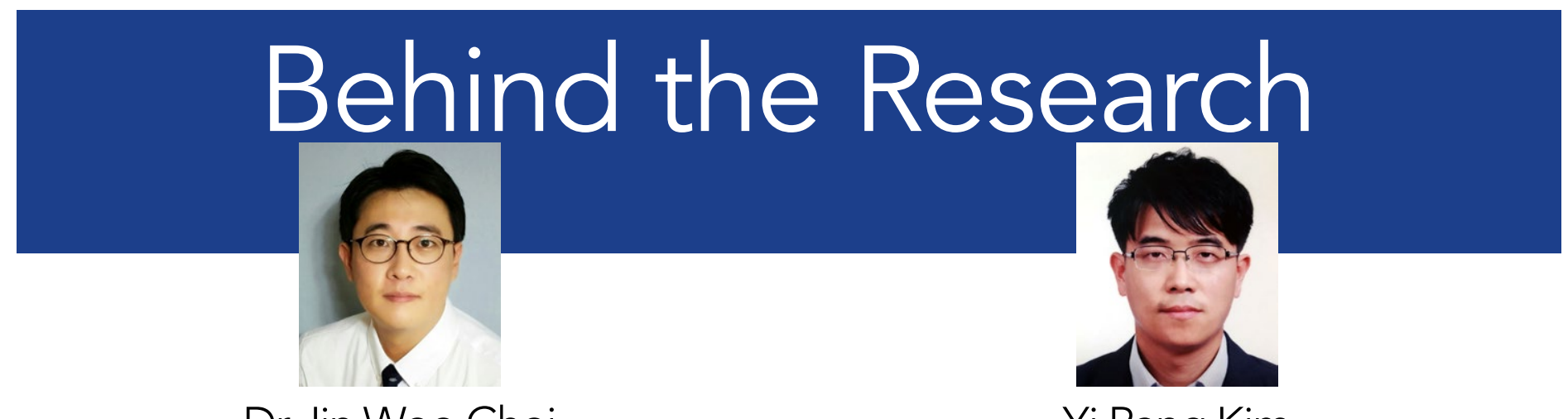

Dr Jin Woo Choi

Yi Rang Kim

E: jinwoo.ch@khu.ac.kr T: $+821040473070 \quad$ W: www.drugdiscovery.co.kr Oncocross, Ltd: E: 99yirang@gmail.com T: +821026828270 W: www.oncocross.com

Research Objectives

The research of Dr Jin Woo Choi \& Yi Rang Kim aim to find novel targets for the regulation of cancer metastasis using novel chemical and gene therapies.

\section{Detail}

Dr Jin Woo Choi

armacology

College of Pharmacy,

Kyung Hee University,

Republic of Korea.

Bio

Dr Jin Woo Choi's PhD degree focused on cancer genetics at Seoul National University. After his research fellow training at Harvard Medical School, Dr Choi became Associate Professor within the Pharmacology Department at Kyung Hee University. His team's current research intere is focused on finding novel targets for the regulation of cancer metastasis andinterv trained Asan Medical Center And he recis a his PhD deoge about photodymic therapy-based bio-optical research

\section{Funding}

This work was supported by National R\&D Program for Cancer Control, Ministry of Health and Welfare, Republic

\section{Collaborators}

Chang Wook Jeong, Department of Urology, Seoul National University Hospital, Seoul, 03080, Republic of Korea

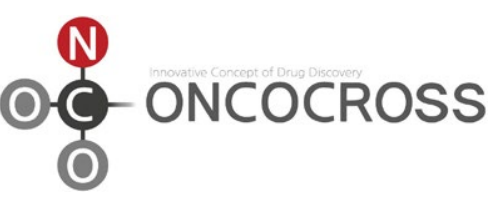

\section{References}

Kim, Y.R., Kim, S., Choi, J.W., Choi, S.Y., Lee, S.H., Kim, H., Hahn, S.K., Koh, G.Y. and Yun, S.H., 2015. Bioluminescen Theranostics, 5(8), p.805.

Kim, Y.R., Kim, J.K. and Choi, J.W., 2017. Fluorescent cellselective ablation using an adaptive photodynamic method. Chemical Communications, 53(92), pp.12434-12437.

Kim, Y.R., Yoo, J.K., Jeong, C.W. and Choi, J.W., 2018 Selective killing of circulating tumor cells prevents metastasis $11(1)$, p.114

Seyfried, T.N. and Huysentruyt, L.C., 2013. On the origin of cancer metastasis. Critical Reviews in Oncogenesis, 18(1-2), p.43.

\section{Personal Response}

How can resonance energy transfer be combined with photodynamic therapy to prevent metastasis?

II Resonance energy transfer is the key mechanism to eradicate GFP cells selectively. By killing GFP-expressing CTCs, we demonstrated that CTCs might be an effective therapeutic target to significantly delay distant metastasis directly suggests CTCs are a core seed to be metastasised into secondary organs. However, these data do not suggest the immediate clinical application of the PDT method examined in this study. Advancements in the field of molecular diagnostics have made it possible to use or molecular or ted photesensitisers in diverse bistisers or molecular-targeted photosensitisers in diverse biological
fields, including not only CTCs-targeted therapy but also cancer stem cell-targeted therapy. 